\title{
Long-Term Effects of Unsaturated Fatty Acid Dominance on the Release of Free Radicals in the Rat
}

\author{
E. TOMSITS, K. RISCHÁK, AND L. SZOLLÁR \\ Institute of Pathophysiology, Semmelweis University of Medicine, Budapest, Hungary
}

\begin{abstract}
The aim of this study was to investigate the effects of an abundance of unsaturated fatty acids, hyperoxia, and vita$\min \mathrm{E}$ on free radical formation in vitamin E-deficient rats. The excess of unsaturated fatty acids was achieved by i.v. administration of a lipid emulsion (Intralipid). To assess free radical formation, we measured the autooxidative susceptibility of red blood cells (AOS) and the thiobarbituric acid reacting substrates (TBARS) in LDL and HDL. Intralipid significantly increased all the measured parameters compared with controls (AOS: $1385 \pm 73$ versus $1056 \pm 55$; LDL-TBARS: $4955 \pm 422$ versus $1050 \pm 33$; HDL-TBARS: $6855 \pm 573$ versus $1033 \pm 26 \mathrm{nmol}$ TBARS/mmol). Hyperoxia alone increased AOS more than Intralipid alone, whereas LDL- and HDL-TBARS concentrations were affected less by hyperoxia than lipid emulsion. The combination of hyperoxia and Intralipid was most effective in raising all measured parameters (AOS: $2285 \pm 141$; LDLTBARS: $6716 \pm 318$; HDL-TBARS: $14614 \pm 1000 \mathrm{nmol}$ TBARS/mmol). Vitamin E completely prevented the in-
\end{abstract}

ABSTRACT

crease in AOS, LDL-TBARS, and HDL-TBARS without fully reversing the increase in free radical formation caused either by Intralipid or by the combination of hyperoxia and Intralipid. These findings suggest that vitamin E supplementation is beneficial to counter increased free radical formation, such as that in response to hyperoxic attacks or lipid-containing parenteral nutrition, which is frequently encountered in the treatment of premature infants. (Pediatr Res 36: 278-282, 1994)
Abbreviations
AOS, autooxidative susceptibility
CH, cholesterol
PUFA, polyunsaturated fatty acid
RBC, red blood cell
TBARS, thiobarbituric acid reacting substance
TG, triglyceride
UFA, unsaturated fatty acid

Clinical studies $(1,2)$ have shown that the administration of lipid emulsions containing an excess of UFA to immature infants causes a higher frequency of disorders thought to be related to increased free radical formation.

Paradoxically, in vitro studies indicate that an excess of certain UFA in the cellular environment can prevent the cytotoxic effects caused by enhanced free radical formation (3-5).

The primary aim of this study was to evaluate the possibility that an abundance of UFA created by the infusion of a lipid emulsion alone or in combination with hyperoxia can influence peroxidation and increase free radical activity that can be ameliorated by the coadministration of the antioxidant vitamin E. Accordingly, we exposed young male rats maintained on a vitamin E-de-

Received April 22, 1994; accepted March 21, 1994.

Correspondence and reprint requests: Erika Tomsits, M.D., Institute of Pathophysiology, Semmelweis University of Medicine, P.O. Box 370, H-1445 Budapest, Hungary.

Supported in part by Grants ETK 7/451 of the Ministry of Health, OTKA 185 and OTKA 1057 of the National Scientific Research Foundation, and ETT 191 of the Ministry of Public Welfare. ficient diet to a prolonged infusion of lipid emulsion alone or in combination with hyperoxia, although this model may not be ideal to reproduce the conditions encountered by premature infants receiving parenteral therapy. The results of this study indicate nonetheless that inclusion of vitamin $\mathrm{E}$ in the therapeutic regimen attenuates the undesirable effects of excessive free radical formation.

\section{METHODS}

A total of 75 male Wistar rats weighing from 180 to 200 $\mathrm{g}$ (aged $6 \mathrm{wk}$ ) were kept in metabolic cages and were fed a vitamin E-deficient diet (LATI, Gödöllô, Hungary) ad libitum for $35 \mathrm{~d}$ before the start of the study and throughout the experiment, in an attempt to mimic the lower vitamin $E$ levels known to occur in premature infants (6). On the 35th d, a catheter was implanted into the inferior cava through the tail vein, using surgical level anesthesia $(25 \mathrm{mg} / \mathrm{kg}$ pentobarbital, EGIS, Budapest, Hungary). We set up eight groups randomly. Four groups of animals were kept in normoxic conditions and four in hyperoxic conditions (fraction of inspired oxygen: 70\%). Within the 
four normoxic groups, one group was infused with Intralipid 20\% (Kabi Vitrum, Stockholm, Sweden) for $16 \mathrm{~h} / \mathrm{d}$ through the venous cannula (group L: $n=8$ ). The second group received lipid and vitamin E (group LE: $n=10$ ). The third group received only vitamin $\mathrm{E}$ (group $\mathrm{E}: n=$ 10 ), and the fourth served as the control (group C: $n=$ $10)$. The same experimental design was followed in the hyperoxic group (group OL: $n=10$; group OLE: $n=8$; group OE: $n=9$; and group O: $n=10$ ). All groups that were not infused with lipid received the same volume of sterile saline. When setting the dose of i.v. lipid emulsion, we followed the proposals of the Society of American Pediatricians (7), i.e. $0.5 \mathrm{~g} / \mathrm{kg} / \mathrm{d}$ in the beginning, reaching $3 \mathrm{~g} / \mathrm{kg} / \mathrm{d}$ at the end in a gradually increasing proportion. So, the total TG intake was 7-9 $\mathrm{g}$ in $9 \mathrm{~d}$, depending on the weight of the animals. The groups administered vitamin $E$ received $20 \mathrm{mg} / \mathrm{kg}$ tocopherol acetate (vitamin E, Gedeon Richter Pharmaceutical Co., Budapest, Hungary) through a gastric tube. Our experiment was approved by the institutional committee for animal research. The experimental protocol is summarized in Figure 1.

$\mathrm{PO}_{2}$ was determined from mixed blood drawn from the tail at both the beginning and the end of the study (biologic microanalyzer OP-210/3; Radelkis, Budapest, Hungary). At the end of the experiment, on the 44th d, blood was collected through the femoral artery into heparinized tubes from all animals under pentobarbital $(25 \mathrm{mg} / \mathrm{kg})$ anesthesia. Blood samples were immediately centrifuged at $1500 \times g$ for $30 \mathrm{~min}$ and then analyzed. Total $\mathrm{CH}$, total TG, LDL CH, and HDL CH levels were determined by Boehringer enzymatic kit (Cholesterol Monotest 237574, CHOD-PAP method; Triglycerides 701904, GPO-PAP method; Boehringer-Mannheim GmbH, Mannheim, Germany). Because the $20 \%$ Intralipid solution increased

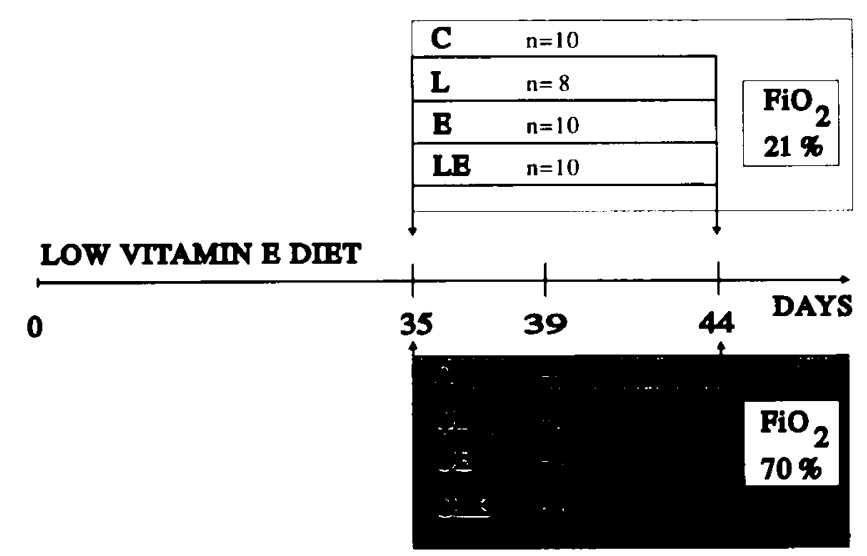

Figure 1. Experimental protocol. Animals were kept on a low vitamin E diet throughout the experiment. On $\mathrm{d} 35$ of the diet, Intralipid infusion was started and administered in a progressively increasing dose until d 39. From d 39 until d 44 , Intralipid $20 \%$ was given in a dose of $3 \mathrm{~g} / \mathrm{kg} / \mathrm{d}$. From $\mathrm{d} 35$, half of the animals were in normoxic conditions [fraction of inspired oxygen $\left(\mathrm{FiO}_{2}\right) 21 \%$ ] and the other half were in hyperoxic conditions $\left(\mathrm{FiO}_{2} 70 \%\right)$. Names of groups: $\mathrm{C}$ : control; L: lipid alone; $\mathrm{E}$ : vitamin E; LE: lipid and vitamin E; O: hyperoxia alone; OL: hyperoxia and lipid; OE: hyperoxia and vitamin E; and OLE: hyperoxia, lipid, and vitamin E. $n$ represents the number of animals in each group. plasma lipoprotein concentration, we always corrected the measured plasma LDL-TBARS and HDL-TBARS concentrations by using the following equation:

$\left[\mathrm{LDL}-\mathrm{TBARS}_{\mathrm{c}}\right]=\frac{\left[\text { PlasmaLDL }_{\mathrm{B}}\right]}{\left[\text { PlasmaLDL }_{\mathrm{A}}\right]} \times\left[\mathrm{LDL}-\right.$ TBARS $\left._{\mathrm{M}}\right]$

where $\mathrm{C}=$ corrected value, $\mathrm{B}=$ before lipid infusion, $\mathrm{A}$ $=$ after lipid infusion, and $\mathrm{M}=$ measured value.

Vitamin E concentrations of plasma specimens and lipid emulsion were measured by a spectrophotometric method (8). Free radical production was monitored by measuring the quantity of RBC (9) as well as plasma LDL and HDL products (10) reacting with thiobarbituric acid (TBARS). Stock's method (9) to measure AOS is based on the generation under oxidative stress of malonyldialdehyde, a secondary breakdown product of lipid peroxides. We incubated the RBC suspensions with constant $\mathrm{Hb}$ concentration in hydrogen peroxide solution for $2 \mathrm{~h}$. Then the released TBARS was estimated by spectrophotometry. Yagi's method (10) measures the released TBARS from plasma HDL and LDL. These methods are widely used to evaluate the quantity of lipid peroxidation, which is an autocatalytic free radical-mediated process whereby PUFA in cell membranes undergo degradation to form lipid hydroperoxides, which easily decompose to produce end products of lipid peroxidation (11).

All data are presented as means \pm SEM. Statistical evaluations were performed by using STATGRAPHICS 4.0 (STSC Inc., Rockville, MD). $p$ values of less than 0.05 were considered significant. Significant differences can be detected on the figures if the $95 \%$ confidence intervals of any group did not overlap.

\section{RESULTS}

The $\mathrm{Po}_{2}$ values determined from mixed blood samples of the tail under normoxic and hyperoxic conditions were found to be $72.8 \pm 8.6$ and $135.8 \pm 18.6 \mathrm{~mm} \mathrm{Hg}$, respectively. Administration of vitamin $\mathrm{E}$ at the given dose caused a significant rise in total plasma tocopherol levels (group C: $0.157 \pm 0.008 \mathrm{mmol} / \mathrm{L}$; group E: $0.311 \pm 0.0015$ $\mathrm{mmol} / \mathrm{L}$ ). The vitamin $\mathrm{E}$ content of the Intralipid solution was $1.13 \pm 0.33 \mathrm{~g} / \mathrm{dL}$; thus, animals could have gained a maximum of $0.66 \mathrm{mg} / \mathrm{d}$ from this solution. It did not cause a further significant change in vitamin $E$ concentration in the plasma of vitamin E-supplemented groups (group LE: $0.336 \pm 0.017 \mathrm{mmol} / \mathrm{L}$; group $\mathrm{LE}$ versus group $\mathrm{E}$ : NS). The 9-d period of Intralipid administration in normoxia had no significant effect on the animals' dietary intake and body weight. Hyperoxia caused a statistically insignificant increase in dietary intake and no change in body weight compared with controls.

In groups receiving Intralipid, total TG and total $\mathrm{CH}$ concentrations increased significantly by the end of the study $\left(\mathrm{TG}_{\mathrm{C}}: 0.52 \pm 0.18 ; \mathrm{TG}_{\mathrm{L}}: 5.75 \pm 0.61 ; \mathrm{TG}_{\mathrm{OL}}: 6.27 \pm\right.$ $1.34 ; \mathrm{TG}_{\mathrm{OLE}}: 5.43 \pm 0.72 ;$ and $\mathrm{TG}_{\mathrm{LE}}: 4.67 \pm 0.84$ $\mathrm{mmol} / \mathrm{L} . \mathrm{CH}_{\mathrm{C}}: 1.28 \pm 0.65 ; \mathrm{CH}_{\mathrm{L}}: 1.73 \pm 0.72 ; \mathrm{CH}_{\mathrm{OL}}$ : 
$1.98 \pm 0.86 ; \mathrm{CH}_{\mathrm{OLE}}: 1.75 \pm 1.03$; and $\mathrm{CH}_{\mathrm{LE}}: 1.87 \pm 0.45$ $\mathrm{mmol} / \mathrm{L}$ ). Plasma total $\mathrm{CH}$ concentrations reflected the increase of both LDL $\mathrm{CH}$ and HDL CH.

AOS (Fig. 2) significantly increased in normoxic animals receiving lipid infusion $(C: 1056 \pm 55 ; \mathrm{L}: 1385 \pm 73$; and LE: $1131 \pm 106 \mathrm{nmol}$ TBARS/g Hb). Hyperoxia alone could significantly elevate AOS versus control $(O$ : $2173 \pm 132$; C: $1056 \pm 55 \mathrm{nmol}$ TBARS/g Hb). Hyperoxia and lipid infusions together resulted in higher AOS values than hyperoxia alone (OL: $2639 \pm 211$ versus O: $2173 \pm$ $132 \mathrm{nmol}$ TBARS/g Hb). Vitamin E by itself decreased AOS in normoxic animals compared with controls $(\mathrm{C}$ : $1056 \pm 55$; E: $887 \pm 42 \mathrm{nmol} \mathrm{TBARS/g} \mathrm{Hb}$ ). A combination of hyperoxia and vitamin $E$ administration did not result in a significant difference from control values (OE: $890 \pm 59 \mathrm{nmol} \mathrm{TBARS} / \mathrm{g} \mathrm{Hb}$ ) (Fig. 2).

Lipid infusion significantly increased both LDLTBARS (Fig. 3) and HDL-TBARS (Fig. 4) concentrations compared with controls (LDL-TBARS ${ }_{\mathrm{L}}: 4955 \pm$ 422 versus $\mathrm{LDL} \mathrm{TBARS}_{\mathrm{C}}: 1050 \pm 33 \mathrm{nmol}$ TBARS $/ \mathrm{mmol}$ LDL and HDL-TBARS ${ }_{\mathrm{L}}: 6855 \pm 573$ versus HDLTBARS $_{C}: 1033 \pm 26 \mathrm{nmol}$ TBARS/mmol HDL). Hyperoxia alone led to an even higher increase $\left(\right.$ LDL-TBARS ${ }_{\mathrm{O}}$ : $1651 \pm 62 \mathrm{nmol}$ TBARS/mmol LDL and HDL-TBARS ${ }_{\mathrm{O}}$ : $1381 \pm 42 \mathrm{nmol}$ TBARS/mmol HDL) (Figs. 3 and 4 ). Hyperoxia and lipid infusion together resulted in the

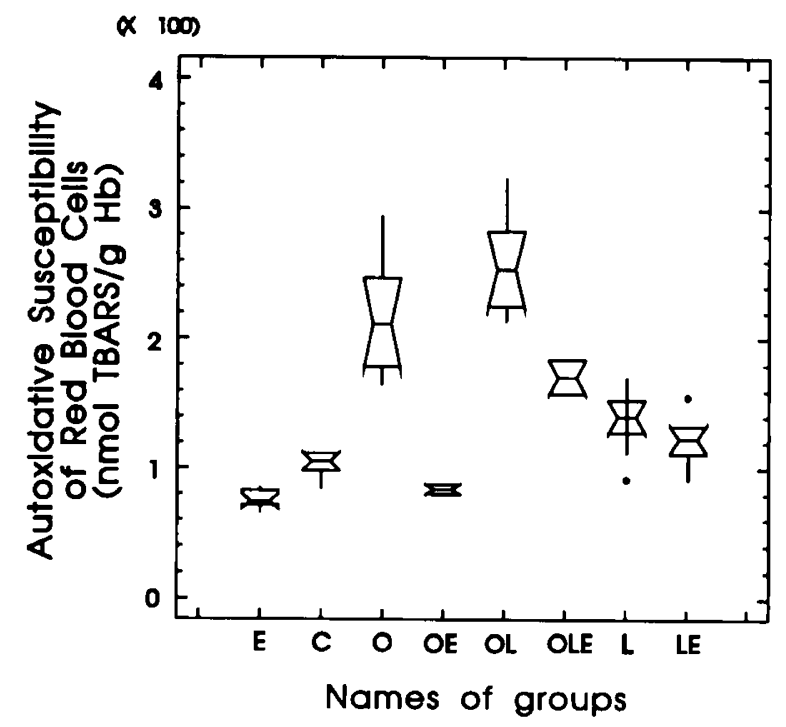

Figure 2. Changes of AOS of RBC. Hyperoxia caused a significant elevation in plasma TBARS concentrations $(O)$. Vitamin E completely prevented this effect $(E)$. Lipid together with hyperoxia resulted in the highest TBARS levels $(O L)$. Although vitamin E significantly reduced the level of TBARS $(O L E)$, it could not totally eliminate the effect of Intralipid $(L E)$. The territory between the upper and the lower horizontal line of a group's symbol refers to the $95 \%$ confidence interval of the parameters examined. The horizontal line in between represents the median value. Error bars are also shown as upper and lower vertical lines on a symbol of a group. The description method we used, particularly when comparing the groups with one another, clearly shows the significant differences between any two groups, i.e. the difference is significant $(p<0.05)$ if the $95 \%$ confidence intervals of the two groups do not overlap. The farther the confidence intervals are from one another, the more significant the difference is between the two groups. Closed circles represent outranging data.

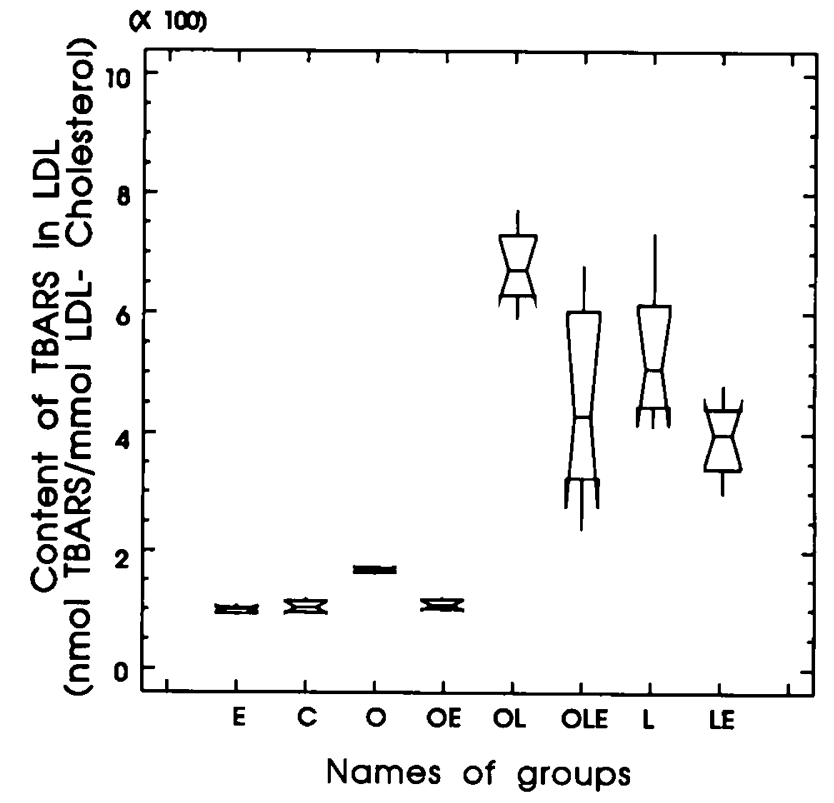

Figure 3. Alterations of LDL-TBARS concentrations in several groups. Intralipid administration caused a significant elevation in LDLTBARS concentrations $(L)$. The combination of Intralipid and vitamin E caused a significant decrease $(L E)$. Hyperoxia together with lipid led to the highest LDL-TBARS increase $(O L)$, and vitamin E significantly decreased it to the level $(O L E)$ that was brought on by lipid alone $(L)$.

highest LDL-TBARS concentration (LDL-TBARS ${ }_{\mathrm{OL}}$ : $6716 \pm 318 \mathrm{nmol}$ TBARS $/ \mathrm{mmol} \mathrm{LDL}$ and HDLTBARS $_{\mathrm{OL}}: 14614 \pm 1000 \mathrm{nmol}$ TBARS/mmol HDL).

Vitamin $\mathrm{E}$ administration caused a decline both in LDL-TBARS and HDL-TBARS concentrations in hyperoxia (LDL-TBARS ${ }_{\mathrm{O}}: 1651 \pm 62$ versus LDLTBARS $_{\mathrm{OE}}: 1137 \pm 37 \mathrm{nmol}$ TBARS/mmol LDL and HDL-TBARS $_{\mathrm{O}}: 1381 \pm 42$ versus HDL-TBARS OE $_{1}: 1149$

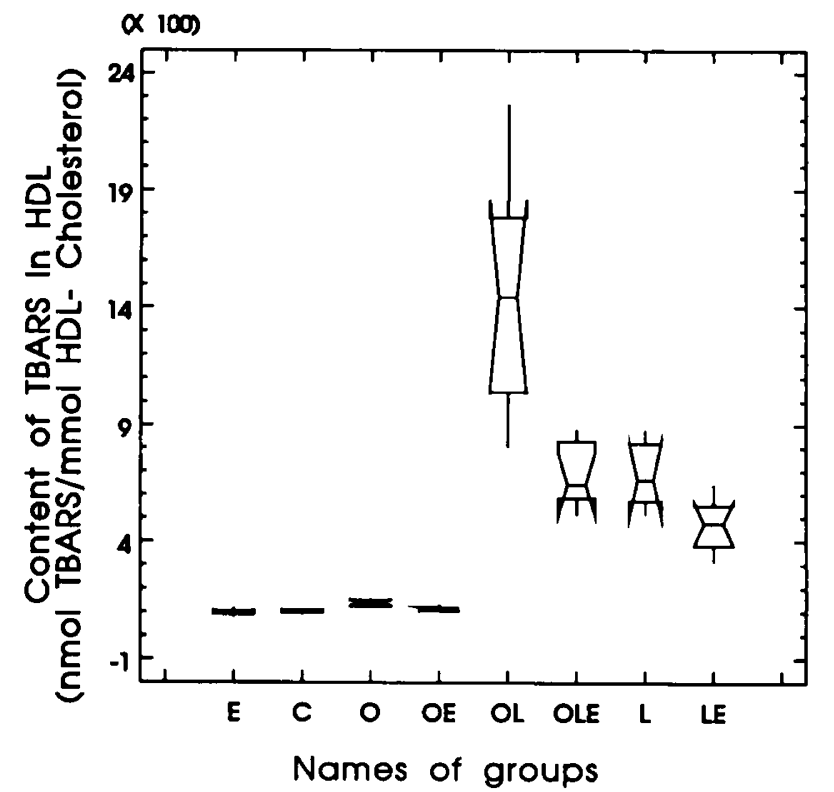

Figure 4. Changes in HDL-TBARS concentrations in different groups. Hyperoxia $(O)$, as well as Intralipid alone $(L)$, significantly increased HDL-TBARS concentration. Vitamin E completely prevented the effect of hyperoxia $(O E)$, but it could not fully eliminate the high HDLTBARS level due to the combined effect of hyperoxia and lipid (OLE). 
$\pm 32 \mathrm{nmol}$ TBARS/mmol HDL). Lipid infusion and vitamin $E$ administration together resulted in a decrease in TBARS concentrations compared with the lipid infusion without vitamin $\mathrm{E}$ (LDL-TBARS ${ }_{\mathrm{LE}}: 3946 \pm 105$ versus LDL-TBARS $_{\mathrm{L}}: 4955 \pm 422 \mathrm{nmol}$ TBARS/mmol LDL and HDL-TBARS $_{\mathrm{LE}}: 5034 \pm 263$ versus HDL-TBARS $_{\mathrm{L}}: 6855$ $\pm 573 \mathrm{nmol}$ TBARS/mmol HDL). Vitamin E administration to hyperoxic and lipid-infused groups led to a significant fall in both LDL-TBARS and HDL-TBARS concentrations (LDL-TBARS ${ }_{\mathrm{OLE}}: 4092 \pm 142$ versus LDLTBARS $_{\mathrm{OL}}: 6716 \pm 318 \mathrm{nmol}$ TBARS/mmol LDL and HDL-TBARS $_{\text {OLE }}: 6293 \pm 796$ versus HDL-TBARS $_{\mathrm{OL}}$ : $14614 \pm 1000 \mathrm{nmol}$ TBARS/mmol HDL) (Figs. 3 and 4).

\section{DISCUSSION}

A good deal of information has accumulated concerning the effects of the overload or deficiency of UFA on the balance of free radical formation and elimination (12-16). Membrane lipids are quite often exposed to nonenzymatic, oxidative processes mediated by free radicals, which result in the destruction of cellular and tissue structures (17). The same oxidative processes are thought to be responsible for several pathologic alterations. Clinical studies have suggested that the presence of excess quantities of free radicals could increase the prevalence of certain diseases, which are thus thought to be mediated by free radicals, e.g. aging, atherosclerosis, retrolental fibroplasia, and bronchopulmonary dysplasia $(1,2,18-21)$. The extent of membrane lipid peroxidation depends on the quantity and quality of the unsaturated or saturated forms of lipids both as membrane components and as constituents of the cellular environment (22-24). It has been demonstrated that in epithelial cell cultures the administration of PUFA decreases the cytotoxic effects caused by hyperoxia, while at the same time the level of TBARS is also decreased $(3,4)$. Other data in the literature show that the oxidative damage of in vitro endothelial cells can be reduced by the administration of saturated and monounsaturated fatty acids, even though opposite effects have been detected in the presence of certain PUFA (5). Wispe et al. (25) in their studies observed significantly increased lipid peroxidation when using parenteral lipid emulsions, and Pitkänen $(26,27)$ proposed that adverse effects of parenteral lipid emulsion are partially caused by free radicals. There are several reports about the role of $\alpha$-tocopherol acetate (vitamin E) in the protection of either hyperoxia- or UFA dominance-caused lipid peroxidation (28-31).

In our series of investigations, we were seeking a model for a frequently found condition observed in premature infants exposed to hyperoxic attacks and receiving lipid-containing parenteral nutrition. We evaluated the in vivo effects of an abundance of UFA on free radical production in a hyperoxic condition with and without administration of vitamin $\mathrm{E}$, a well-known antioxidant. The free radical production was characterized by two different TBARS assays of RBC, LDL, and HDL. Al- though, the TBARS assay has previously been criticized and there are other methods to measure lipid peroxidation (32), it is still an accepted and widely used procedure (33-35).

The Intralipid $20 \%$ solution did not affect significantly the vitamin $E$ level of the animals. All the parameters we checked showed that UFA excess causes a significant increase in the concentration of TBARS of the RBC and plasma LDL and HDL levels, which are considered the characteristic signs of increased free radical production $(8,36)$. Our findings therefore seem to support clinical observations that total parenteral nutrition with lipid emulsions leads to increased formation of free radicals. We found that vitamin $E$ administration prevents the effects of free radical increase caused by hyperoxia, whereas it could only attenuate free radical production as a result of UFA intake. Moreover, our results indicate that the PUFA of the Intralipid $20 \%$ cause increased lipid peroxidation of RBC membranes. A possible explanation for this may be that long-term administration of PUFA leads to their integration into plasma membranes and that such PUFA surplus in the cellular environment does not inhibit lipid peroxidation of plasma membranes in vivo.

Based on our findings, we conclude that either hyperoxia or the administration of lipid emulsion can cause a significant increase in the production of free radicals.

Vitamin E supplementation prevents the rise in the AOS of RBC under hyperoxia, because no significant changes were detected between the hyperoxic group with vitamin $\mathrm{E}$ and the normoxic group. Furthermore, vitamin E administration could not fully eliminate the significant increase in free radical production caused by lipid infusion. Intralipid along with hyperoxia significantly increased lipid peroxidation, and this increase was higher than that caused by either the administration of lipid emulsion or hyperoxia.

The use of otherwise healthy young adult animals, however, may not allow strict conclusions for sick humans' antioxidant defense. Nonetheless, our results suggest that vitamin $\mathrm{E}$ administration may represent an important therapeutic intervention in the treatment of sick premature newborns, who often suffer from hyperoxic attacks and require lipid-containing parenteral nutrition and who are more sensitive to free radical formation than adult patients.

\section{REFERENCES}

1. Cooke RWI 1991 Factors associated with chronic lung disease in preterm infants. Arch Dis Child 66:776-779

2. Hammerman C, Aramburo MJ 1988 Decreased lipid intake reduces morbidity in sick premature neonates. J Pediatr 113:1083-1087

3. Dennery PA, Kramer CM, Alpert SE 1989 Thiobarbiturate-reactive substances and activation of the arachidonic acid cascade in cultured rabbit tracheal epithelial cells exposed in vitro to hyperoxia. Am Rev Respir Dis 139:42a(abstr)

4. Dennery PA, Kramer CM, Alpert SE 1990 Effect of fatty acid profiles on the susceptibility of cultured rabbit tracheal epithelial cells to hyperoxic injury. Am J Respir Cell Mol Biol 3:137-144

5. Hart CM, Tolson JK, Block ER 1991 Supplemental fatty acids alter lipid peroxidation and oxidant injury in endothelial cells. Am J Physiol 260:L481L488 
6. Gutcher GR, Raynor WJ, Farrell PM 1984 An evaluation of vitamin E status in premature infants. Am J Clin Nutr 40:1078-1089

7. American Academy of Pediatrics Committee on Nutrition 1981 Use of intravenous fat emulsion in pediatric patients. Pediatrics 68:738-743

8. Quaife ML, Serimshaw NS, Lowry OH 1967 Gyorgy P, Pearson WN (eds) The Vitamins: Chemistry, Physiology, Pathology, Methods, Vol VI. Academic Press, New York, pp 299-301

9. Stocks J, Offerman EL, Modell CB. Dormandy TL 1972 The susceptibility to autoxidation of human red cell lipids in health and disease. Br J Haemato 23:713-724

10. Yagi K 1976 A simple fluorometric assay for lipoperoxide in blood plasma. Biochem Med 15:212-216

11. Knight JA, Pieper RK, McClellan L 1988 Specificity of the thiobarbituric acid reaction: its use in studies of lipid peroxidation. Clin Chem $34 \cdot 12 \cdot 2433-2438$

12. Marinier E 1991 Les emulsions lipidiques en nutrition parenterale. Gastroenterol Clin Biol 15:956-967

13. Scaccini C, Nardini M, D’Auquino M, Gentili V, Di Felici M, Tomassi G 1992 Effect of dietary oils on lipid peroxidation and on antioxidant parameters of rat plasma and lipoprotein fractions. J Lipid Res 33:627-633

14. Berry EM 1992 The effects of nutrients on lipoprotein susceptibility to oxidation. Curr Opin Lipid 3:5-11

15. Sosenko IRS, Innis SM, Frank L 1988 Polyunsaturated fatty acids and protection of newborn rats from oxygen toxicity. J Pediatr 112:630-637

16. Sosenko IRS, Innis SM, Frank L 1991 Intralipid increases lung polyunsaturated fatty acids and protects newborn rats from oxygen toxicity. Pediatr Res 30:413-417

17. Horton AA, Fairshurst S 1987 Lipid peroxidation and mechanisms of toxicity Crit Rev Toxicol 18:72-79

18. Niki E, Yamamoto Y, Komuro E, Sato K 1991 Membrane damage due to lipid oxidation. Am J Clin Nutr 53:201S-205S

19. Karmazsin L, Olah VA, Balla Gy, Makay A 1990 Serum antioxidant activity in premature babies. Acta Paediatr Hung 30:217-224

20. Frank L, Sosenko IRS 1991 Failure of premature rabbits to increase antioxidant enzymes during hyperoxic exposure: increased susceptibility to pulmonary oxygen toxicity compared with term rabbits. Pediatr Res 29:292-296

21. Jamieson DD 1991 Lipid peroxidation in brain and lungs from mice exposed to hyperoxia. Biochem Pharmacol 41:749-756
22. Burns A, Lin Y, Gibson R, Jamieson D 1991 The effect of a fish oil enriched diet on oxygen toxicity and lipid peroxidation in mice. Biochem Pharmacol 42:1353-1360

23. Kehrer JP, Autor AP 1978 The effect of dietary fatty acids on the composition of adult rat lung lipids: relationship to oxygen toxicity. Toxicol Appl Pharmacol 44:423-430

24. Spitz DP, Kinter MT, Kehrer JP, Roberts RJ 1992 The effect of monosaturated fatty acids and polyunsaturated fatty acids on oxygen toxicity in cultured cells. Pediatr Res 32:366-372

25. Wispe JR, Knight M, Roberts RJ 1986 Lipid peroxidation in newborn rabbits: effects of oxygen, lipid emulsion, and vitamin E. Pediatr Res 20:505-510

26. Pitkänen OM 1992 Peroxidation of lipid emulsions: a hazard for the premature infant receiving parenteral nutrition? Free Radic Biol Med 13:239-245

27. Pitkänen O, Holmann M, Anderson SM 1991 Generation of free radicals in lipid emulsion used in parenteral nutrition. Pediatr Res 29:56-69

28. Janero DR 1991 Therapeutic potential of vitamin $E$ against myocardial ischemic-reperfusion injury. Free Radic Biol Med 10:315-324

29. Esterbauer H, Dieber-Rotheneder M, Striegl G, Waeg G 1991 Role of vitamin $E$ in preventing the oxidation of low-density lipoprotein. Am J Clin Nutr $53: 314 \mathrm{~S}-21 \mathrm{~S}$

30. Negre-Salvayre A, Reaud V, Hariton C, Salvayre S 1991 Protective effect of $\alpha$-tocopherol, ascorbic acid and rutin against peroxidative stress induced by oxidized lipoproteins on lymphoid cells lines. Biochem Pharmacol 42:450-453

31. Parker L 1991 Protective role of vitamin E in biological systems. Am J Clin Nutr 53:1050S-1055S

32. Wispe JR, Bell EF, Roberts RJ 1985 Assessment of lipid peroxidation in newborn infants and rabbits by measurements of expired ethane and pentane: influence of parenteral lipid infusion. Pediatr Res 19:374-379

33. Pryor WA 1989 On the detection of lipid hydroperoxides in biological samples. Free Radic Biol Med 7:177-178

34. Kosugi H, Kikugawa K 1989 Potential thiobarbituric acid-reactive substances in peroxidized lipids. Free Radic Biol Med 7:205-207

35. Kosugi H, Poima T, Kikugawa K 1989 Thiobarbituric acid reactive substances from peroxidized lipids. Lipids 24:873-881

36. Dillard CJ, Tappel AL 1989 Lipid peroxidation products in biological tissues. Free Radic Biol Med 7:193-196 